\title{
Development of cosmetic formulations containing glucan polymer of Cassava (Manihot esculenta): stability and sensory analysis
}

\author{
Desenvolvimento de formulações cosméticas contendo o polímero glucan de Mandioca \\ (Manihot esculenta): Estabilidade e análise sensorial
}

\author{
Luisa M. Manço, Daiane G. Mercurio, Maísa O. Melo and Patrícia M. B. G. Maia Campos \\ Faculty of Pharmaceutical Sciences of Ribeirão Preto, University of São Paulo, Av. do Café S/N, Monte Alegre, 14040-903, \\ Ribeirão Preto, SP, Brazil \\ Email: pmcampos@usp.br
}

\begin{abstract}
The aim of this study was the development, rheological behaviour determination, and sensory analysis of cosmetic formulations containing glucan biopolymer (Manihot esculenta), a tensor agent that was proposed to produce an immediate lifting and smoothing effect. For this purpose, formulations were developed and supplemented or not with $4 \%$ of hydrolysed Manihot esculenta tuber extract and submitted to preliminary stability tests. These formulations were evaluated in terms of rheological behaviour over 90 days. Sensory analysis was carried out through a research with 20 cosmetic consumers who answered a questionnaire regarding their perception to the cosmetic qualities. The formulations presented pseudoplastic behavior and were considered stable in the physical stability studies, with the exception of the gel formulation based on Ammonium Acryloyldimethyltaurate/VP Copolymer. The formulations were well evaluated in the sensory parameters. The gel formulations based on Polyacrylamide, C1314 Isoparaffin, and Laureth-7 were stable and presented the best sensory profile in some evaluated parameters, such as spreadability, smoothness and skin moisturizing, and can be considered an appropriate vehicle for formulations containing hydrolysed Manihot esculenta tuber extract.
\end{abstract}

Keywords: glucan biopolymer, stability, sensory properties, cosmetic

\section{Resumo}

O objetivo desse trabalho é o desenvolvimento, avaliação do comportamento reológico e análise sensorial de formulações contendo o biopolímero glucan (Manihot esculenta). Para tal, foram desenvolvidas formulações que foram adicionadas, ou não, de $4 \%$ do extrato hidrolisado da raíz de Manihot esculenta e submetidas a testes preliminares de estabilidade. Essas formulações foram submetidas a análises de comportamento reológico durante 90 dias. A análise sensorial foi realizada em um grupo de 20 consumidoras de cosméticos. As formulações foram aplicadas na região do antebraço das voluntárias e foi aplicado um questionário sobre as percepções das qualidades cosméticas. As formulações apresentaram comportamento pseudoplástico e foram consideradas estáveis nos testes de estabilidade física, exceto a formulação em gel a base de Ammonium Acryloyldimethyltaurate/VP Copolymer. As formulações foram bem avaliadas em seus parâmetros sensoriais. As formulações em gel baseadas em Polyacrylamide (and) C13-14 Isoparaffin (and) Laureth-7 foram estáveis e apresentaram o perfil sensorial mais agradável e, considerando os testes de estabilidade física realizados, pode-se concluir que é um veículo apropriado para formulações contendo o extrato hidrolisado da raíz de Manihot esculenta.

Palavras-chave: Biopolímeros Glucan, Estabilidade, Propriedades sensoriais, cosméticos. 


\section{Introduction}

Aging is a complex process composed of chronological (intrinsic) factors, linked to genetic material, as well as extrinsic factors, linked to ultraviolet radiation, alcohol, smoking, poor diet and harsh environmental conditions $[1,2]$

During aging, qualitative and quantitative changes occur in the skin, including the loss of elasticity, increased wrinkling, and reduction in epidermal thickness and collagen content ${ }^{[3,4]}$. Therefore, the modification of the skin viscoelasticity by cosmetic treatments has been the subject of research and the development of products to improve skin appearance ${ }^{[5,6]}$.

In this context, the hydrolysed Manihot esculenta tuber extract stands out as a new generation of tensor agent. The tridimensional structure quickly adheres, spreads, and forms a viscoelastic film on the skin's surface. This cohesive glucan biopolymer produces an immediate lifting and smoothing effect for both young and mature skin types ${ }^{[7]}$.

The choice of appropriate vehicles for the development of formulations supplemented with this new innovative ingredient is very important, as is the evaluation of their stability and sensory properties. Thus, the aim of this study was the development and rheological behaviour and sensory analysis of cosmetic formulations containing glucan biopolymer (Manihot esculenta).

\section{Methods}

\section{Formulation development}

A total of eight formulations were developed: gels based on Ammonium Acryloyldimethyltaurate/VP Copolymer, Glycerin, Propylene Glycol, Methyldibromo Glutaronitrile, Cyclopentasiloxane, Triethanolamine (F1, V1); gels based on Polyacrylamide (and) C13-14 Isoparaffin (and) Laureth-7, Glycerin, Propylene Glycol, Methyldibromo Glutaronitrile, Cyclopentasiloxane, Triethanolamine (F2, V2); gels based on Ammonium Acryloyldimethyltaurate/VP Copolymer, Glyceryl Stearate (and) PEG-100 Stearate, Glycerin, Propylene Glycol, Methyldibromo Glutaronitrile, Cyclopentasiloxane, Triethanolamine (F3, V3); emulsions based on Polyacrylamide (and) C13-14 Isoparaffin (and) Laureth-7, Glyceryl Stearate (and) PEG-100 Stearate, Glycerin, Propylene Glycol, Methyldibromo Glutaronitrile, Cyclopentasiloxane, Triethanolamine (F4, V4). The formulations were supplemented $(\mathrm{F})$, or not $(\mathrm{V})$,

\section{Introdução}

O envelhecimento é um processo complexo cronológico, relacionado com o material genético (intrínseco) e fatores extrínsecos, ligados com radiações ultravioleta, álcool, fumo, dietas deficientes e condições ambientais severas ${ }^{[1 ; 2]}$

Durante o envelhecimento, mudanças quantitativas e qualitativas ocorrem na pele, como perda da elasticidade, aumento da quantidade de rugas, redução da espessura da epiderme e conteúdo de colágeno ${ }^{[3 ; 4]}$. Assim, a modificação da viscoelasticidade da pele por tratamentos cosméticos está sendo tópico de pesquisas e desenvolvimento de produtos para a melhora da aparência da pele ${ }^{[5 ; 6]}$

Nesse contexto, o extrato hidrolisado da raíz de $M a$ nihot esculenta se destaca como uma nova geração de agentes tensores. A sua estrutura tridimensional se adere rapidamente, espalha e forma um filme viscoelástico sob a superfície da pele. Esse biopolímero glucan coesivo produz um lifting imediato e efeito suavizante para ambas peles maduras e jovens ${ }^{[7]}$.

Assim, é de grande importância a escolha dos veículos apropriados para o desenvolvimento de formulações contendo esse ingrediente ativo inovador e avaliar a sua estabilidade e propriedades sensoriais. Portanto, o objetivo desse trabalho é o desenvolvimento, avaliação do comportamento reológico e análise sensorial de formulações contendo o biopolímero glucan (Manihot esculenta).

\section{Métodos}

\section{Desenvolvimento de formulações}

Oito formulações foram desenvolvidas: géis baseados em Ammonium Acryloyldimethyltaurate/VP Copolymer, Glycerin, Propylene Glycol, Methyldibromo Glutaronitrile, Cyclopentasiloxane, Triethanolamine (F1, V1); géis baseados em Polyacrylamide (and) C13-14 Isoparaffin (and) Laureth-7, Glycerin, Propylene Glycol, Methyldibromo Glutaronitrile, Cyclopentasiloxane, Triethanolamine (F2, V2); emulsões baseadas em Ammonium Acryloyldimethyltaurate/VP Copolymer, Glyceryl Stearate (and) PEG-100 Stearate, Glycerin, Propylene Glycol, Methyldibromo Glutaronitrile, Cyclopentasiloxane, Triethanolamine (F3, V3); emulsões baseadas em Polyacrylamide (and) C13-14 Isoparaffin (and) Laureth-7, Glyceryl Stearate (and) PEG-100 Stearate, Glycerin, Propylene Glycol, Methyldibromo Glutaronitrile, Cyclopentasiloxane, Triethanolamine $(\mathrm{F} 4, \mathrm{~V} 4)$. As formulações foram adicionadas $(\mathrm{F})$, ou 
with $4 \%$ of hydrolysed Manihot esculenta tuber extract and submitted to preliminary stability tests by centrifugation, $\mathrm{pH}$ determination and evaluation of organoleptic characteristics weekly over 90 days.

\section{Rheological behavior study}

The formulations were stored at $25^{\circ} \mathrm{C}, 37^{\circ} \mathrm{C}$ and, $45^{\circ} \mathrm{C}$, for 90 days and submitted to the evaluation of viscosity, flow index, consistency index and thixotropy. The analyses were performed at seven day intervals during this period ${ }^{[8]}$.

Rheological determinations were performed in a DV-III Brookfield rotational rheometer (Stoughton, MA, USA) with a cone-plate configuration. Rheograms and viscosity measurements were made under the following experimental conditions: $25^{\circ} \mathrm{C}, 0.5 \mathrm{~g}$ samples and CP52 spindle. The rheograms obtained were mathematically analysed by the Ostwald Law, and the values of apparent viscosity, flow index and consistency index were obtained. The numeric integration of the rheogram curves provided the loop area hysteresis-thixotropy ${ }^{[9 ; 10]}$

\section{Sensory analysis}

Sensory analysis was carried out through research with cosmetic consumers. A total of 20 healthy female volunteers, mean age 23,3 $\pm 3,4$ years old, participated in this study after giving informed consent. The formulations were applied on the forearm skin of the volunteers, who were asked to answer a questionnaire in order to have information about their perception concerning the cosmetic qualities of sense of touch, spreadability, moisturizing, smoothness, skin sensation ${ }^{[11]}$.

\section{Results and Discussion}

The results obtained in the rheological analysis showed that the formulations have pseudoplastic behavior (flow index $<1$ ), which is of interest in cosmetic products to favour their application and spreadability, conferring a pleasant sensorial experience (Table 1) ${ }^{[12]}$.

The formulations based on Polyacrylamide (and) C1314 Isoparaffin (and) Laureth-7, supplemented (F2), or not (vehicle - V2), with $4 \%$ of hydrolysed Manihot esculenta tuber extract can be considered stable as alterations in the parameters evaluated (Table 1 and 2) after the 90-day-period, with the exception of an increase in apparent viscosity when stored at $45^{\circ} \mathrm{C}$. Considering the rheograms (Figure 1), these formulations were more stable than the others, as they did not show any fluctuation. On the other side, the formulations V1 and F1 não (V), com $4 \%$ do extrato hidrolizado da raíz de $M a$ nihot esculenta, sendo submetidos a testes preliminares de estabilidade por centrífuga, determinação de $\mathrm{pH}$ e avaliações semanais de características organolépticas durante 90 dias.

\section{Análise do comportamento reológico}

As formulações foram armazenadas em $25{ }^{\circ} \mathrm{C}, 37{ }^{\circ} \mathrm{C}$ e, $45^{\circ} \mathrm{C}$, por 90 dias e submetidas para a avaliação do comportamento reológico em intervalos de 7 dias durante o período de estudo ${ }^{[8]}$.

A avaliação reológica foi realizada em um Reômetro rotacional Bookfield modelo DV-III (Soughton, MA, EUA) com configuração cone-placa. Os Reogramas e as medidas de viscosidade foram realizadas nas seguintes condições experimentais: $25^{\circ} \mathrm{C}, 0,5 \mathrm{~g}$ de amostras e spindle CP52. Os reogramas obtidos foram analisados matematicamente pela lei de Ostwald, e os valores de viscosidade aparente, índice de fluxo e índice de consistência foram obtidos. A integração numérica dos reogramas permitiu o calculo da área de histerese - tixotropia ${ }^{[9 ; 10] .}$

\section{Análise Sensorial}

A análise sensorial foi desenvolvida por uma pesquisa com consumidoras atuais de cosméticos. Um total de 20 voluntárias saudáveis, com idade média de 23,3 $\pm 3,4$ anos, participaram nesta fase do estudo, após consentimento das mesmas. As formulações foram aplicadas na região do braço das voluntárias, que preencheram um questionário onde forneceram informações sobre as suas percepções sobre as características cosméticas como sensação ao toque, espalhabilidade, hidratação, maciez e sensação na pele ${ }^{[11]}$.

\section{Resultados e Discussão}

Os resultados obtidos nas análises reológicas mostraram que as formulações estudadas apresentam um comportamento pseudoplático (índice de fluxo $<1$ ), o que é muito interessante em formulações cosméticas, uma vez que esses parâmetros favorecem a aplicação, espalhabilidade e conferindo assim, um sensorial agradável (Tabela 1).

As formulações baseadas em Polyacrylamide (and) C13-14 Isoparaffin (and) Laureth-7, adicionadas (F2), ou não (veículo - V2), com $4 \%$ do extrato hidrolisado da raíz de Manihot podem ser consideradas uma vez que não mostraram flutuações nos parâmetros analisados (Tabela 1 e 2) após o período de 90 dias de estudo, exceto por um aumento de viscosidade aparente quando armazenadas a $45{ }^{\circ} \mathrm{C}$. Considerando os reogramas (Figura 1), essas formulações foram as mais, 
based on Ammonium Acryloyldimethyltaurate/VP Copolymer, Glycerin, Propylene Glycol, Methyldibromo Glutaronitrile, Cyclopentasiloxane, Triethanolamine can be considered unstable according to fluctuations in the rheograms (Fig 1). The formulations V3 and F3 decreased the viscosity when supplemented with hydrolysed Manihot esculenta tuber extract, while the presence of this active ingredient in the formulations V4 and F4 increased the observed viscosity. uma vez que não mostraram nenhum tipo de flutuação. Porém, as formulações V1 e F1 baseadas em Ammonium Acryloyldimethyltaurate/VP Copolymer, Glycerin, Propylene Glycol, Methyldibromo Glutaronitrile, Cyclopentasiloxane, Triethanolamine podem ser consideradas instáveis de acordo com as flutuações observadas nos reogramas (Figura 1). As formulações V3 e F3 também sofreram uma diminuição de viscosidade quando acrescidas com o ingrediente ativo em estudo, e as formulações V4 e F4, em estas mesmas condições, sofreu um aumento de viscosidade.

Table 1/ Tabela 1. Flow index of the formulations after 0, 28, 60 and 90 days, when stored at ambient temperature, or submitted to $37^{\circ} \mathrm{C}$ or $45^{\circ} \mathrm{C}$.

Indice de fluxo das formulações após 0, 28, 60 e 90 dias quando armazenados em temperatura ambiente, ou submetidas a $37^{\circ} \mathrm{C}$ ou $45^{\circ} \mathrm{C}$.

\begin{tabular}{|c|c|c|c|c|c|c|c|c|c|c|c|c|}
\hline \multirow[b]{3}{*}{$\begin{array}{l}\text { Formulation } \\
\text { Formulação }\end{array}$} & \multicolumn{12}{|c|}{ Flow Index/Índice de fluxo } \\
\hline & \multicolumn{4}{|c|}{ Ambient/ Ambiente } & \multicolumn{4}{|c|}{$37^{\circ} \mathrm{C}$} & \multicolumn{4}{|c|}{$45^{\circ} \mathrm{C}$} \\
\hline & T0 & T28 & T60 & T90 & T0 & $\mathbf{T} 28$ & T60 & T90 & T0 & T28 & T60 & T90 \\
\hline $\mathrm{V} 1$ & 0.18 & 0.17 & 0.2 & 0.25 & 0.18 & 0.18 & 0.2 & 0.24 & 0.16 & 0.2 & 0.23 & 0.27 \\
\hline $\mathbf{V} 2$ & 0.24 & 0.23 & 0.23 & 0.23 & 0.24 & 0.23 & 0.22 & 0.22 & 0.23 & 0.22 & 0.23 & 0.24 \\
\hline $\mathbf{V 3}$ & 0.15 & 0.21 & 0.2 & 0.19 & 0.15 & 0.2 & 0.18 & 0.19 & 0.17 & 0.18 & 0.05 & 0.19 \\
\hline V4 & 0.27 & 0.28 & 0.3 & 0.31 & 0.27 & 0.31 & 0.34 & 0.33 & 0.28 & 0.32 & 0.32 & 0.27 \\
\hline F1 & 0.17 & 0.19 & 0.18 & 0.2 & 0.18 & 0.19 & 0.21 & 0.26 & 0.18 & 0.2 & 0.22 & 0.24 \\
\hline F2 & 0.22 & 0.24 & 0.23 & 0.23 & 0.22 & 0.24 & 0.23 & 0.24 & 0.21 & 0.23 & 0.25 & 0.27 \\
\hline F3 & 0.17 & 0.25 & 0.2 & 0.2 & 0.18 & 0.21 & 0.2 & 0.21 & 0.17 & 0.19 & 0.21 & 0.19 \\
\hline F4 & 0.24 & 0.28 & 0.28 & 0.28 & 0.25 & 0.3 & 0.3 & 0.3 & 0.25 & 0.34 & 0.35 & 0.33 \\
\hline
\end{tabular}

Table 2/ Tabela 2. Consistency index of the formulations after 0, 28, 60 and 90 days, when stored at ambient temperature, or submitted to $37^{\circ} \mathrm{C}$ or $45^{\circ} \mathrm{C}$.

Índice de consistência das formulações após 0, 28, 60 e 90 dias, quando armazenados em temperatura ambiente, ou submetidas a $37^{\circ} \mathrm{C}$ ou $45^{\circ} \mathrm{C}$.

\begin{tabular}{|c|c|c|c|c|c|c|c|c|c|c|c|c|}
\hline \multirow[b]{3}{*}{$\begin{array}{l}\text { Formulation } \\
\text { Formulação }\end{array}$} & \multicolumn{12}{|c|}{ Consistency Index/Índice de Consistência } \\
\hline & \multicolumn{4}{|c|}{$\begin{array}{l}\text { Ambient Temperature/ } \\
\text { Temperatura Ambiente }\end{array}$} & \multicolumn{4}{|c|}{ j } & \multicolumn{4}{|c|}{$45^{\circ} \mathrm{C}$} \\
\hline & T0 & T28 & T60 & T90 & T0 & $\mathbf{T} 28$ & T60 & T90 & T0 & $\mathbf{T} 28$ & T60 & T90 \\
\hline $\mathrm{V1}$ & 72148 & 80790 & 68572 & 49119 & 82402 & 84787 & 78001 & 45989 & 91076 & 60959 & 63123 & 65247 \\
\hline $\mathbf{V 2}$ & 45428 & 50607 & 64027 & 67824 & 40866 & 48500 & 57052 & 63239 & 45974 & 51467 & 61101 & 57914 \\
\hline V3 & 82442 & 69915 & 77097 & 87903 & 87044 & 69476 & 82757 & 83539 & 79121 & 87274 & 88444 & 78333 \\
\hline V4 & 17342 & 18930 & 14198 & 18781 & 17180 & 14657 & 13838 & 16444 & 17983 & 13957 & 18142 & 38082 \\
\hline F1 & 84427 & 75593 & 77427 & 71364 & 78080 & 75497 & 70065 & 47231 & 77410 & 68598 & 54173 & 54262 \\
\hline F2 & 45615 & 45823 & 57228 & 65119 & 45837 & 47554 & 54945 & 60198 & 47946 & 48585 & 44605 & 44585 \\
\hline F3 & 48361 & 36659 & 57889 & 71161 & 49848 & 48862 & 58402 & 69016 & 55309 & 56058 & 56850 & 65562 \\
\hline F4 & 23318 & 14529 & 19169 & 25354 & 17996 & 17105 & 18280 & 23691 & 15913 & 12162 & 12600 & 22613 \\
\hline
\end{tabular}



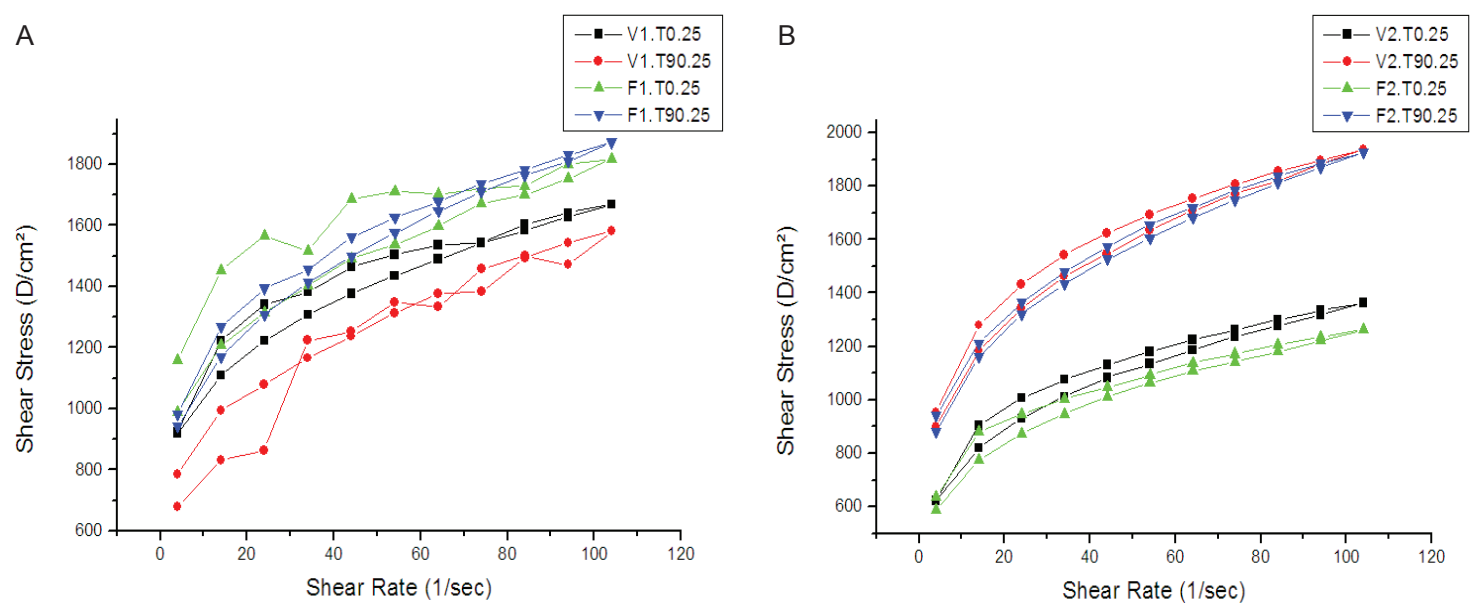

C
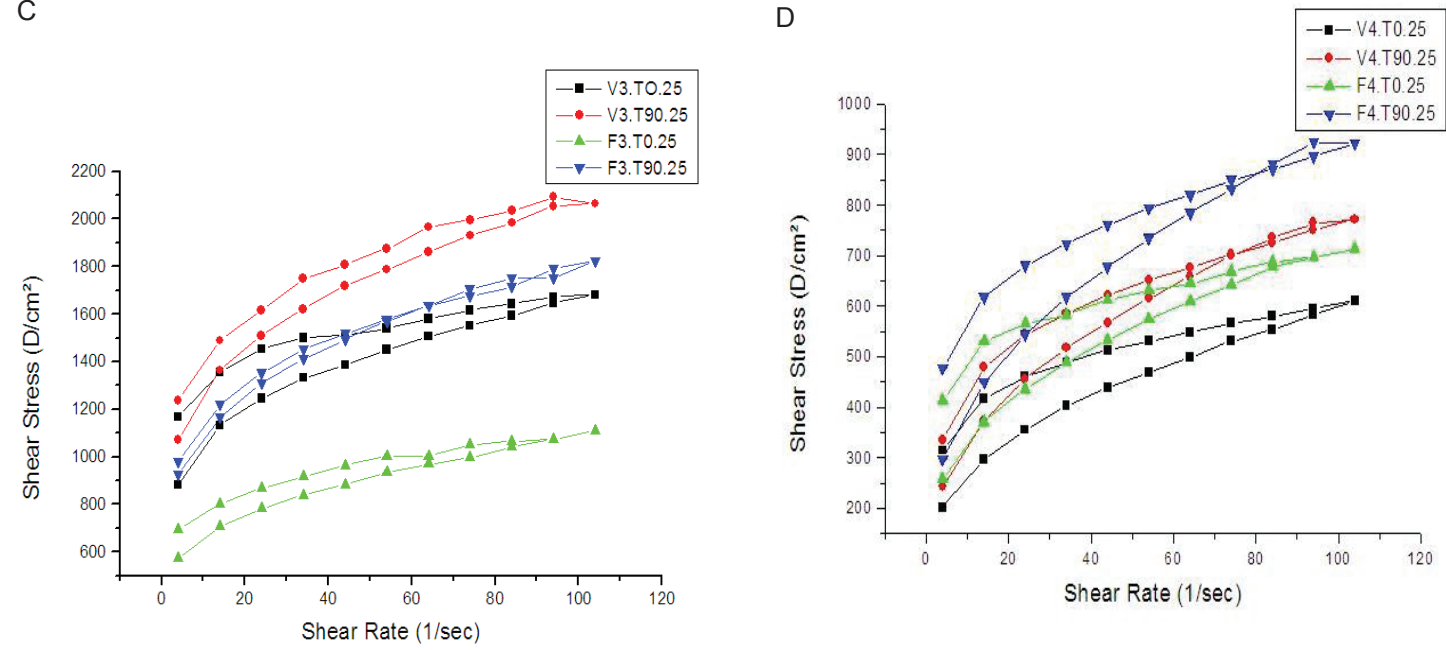

Figure 1/ Figura 1. Rheological behavior of formulations V1 (Vehicle Gel) and F1 (Vehicle Gel + Active Ingredient) (A); V2 (Vehicle Gel) and F2 (Vehicle Gel + Active Ingredient) (B); V3 (Gel cream Vehicle) and F3 (Gel-cream + Active Ingredient) (C); V4 (Vehicle Gel-cream) and F4 (Gel-cream Vehicle + Active Ingredient) (D) at $25^{\circ} \mathrm{C}$ for 90 days.

Comportamento reológico das formulações V1 (gel veículo) e F1 (Gel veículo + Ingrediente ativo) (A); V2 (Gel veículo) e F2 (Gel veículo + Ingrediente ativo) (B); V3 (Gel-creme veículo) e F3 (Gel-creme veículo + Ingrediente ativo) (C); V4 (Veículo gelcreme) e F4 (Gel-creme veículo + Ingrediente ativo) (D) em $25^{\circ} \mathrm{C}$ por 90 dias.

The apparent viscosity is considered the resistance measurement from the material to the flow, as the resistance is directly proportional to the viscosity (Table 2) ${ }^{[13]}$. In pseudoplastic formulations, the apparent viscosity values can be obtained in any point of the rheological curve, and with that, the consistency index values. These values are obtained by the sheer rate versus sheer stress graphic, which is indicated to represent these types of formulations, as this parameter was also considered in this study (Table 3$)^{[14]}$.
A viscosidade aparente é considerada uma medida de resistência do material ao fluxo, sendo que a resistência é diretamente proporcional à viscosidade ${ }^{[13]}$. Em formulações pseudoplásticas, os valores de viscosidade aparente podem ser obtidas em qualquer ponto da curva reológica e deste modo, os valores de índice de consistência, obtidos pelo gráfico de taxa de cisalhamento $\mathrm{x}$ tensão de cisalhamento, que é o indicado para representar este tipo de formulações, sendo que esse parâmetro foi considerado em este estudo. As formulações estudadas não mostraram mudanças significativas em este parâmetro (Tabelas 2 e 3) ${ }^{[14]}$. 
Table 3/ Tabela 3. Apparent viscosity (cP) of the formulations after 0, 28, 60 and 90 days, when stored at ambient temperature, or submitted to $37^{\circ} \mathrm{C}$ or $45^{\circ} \mathrm{C}$.

Viscosidade aparente (cP) das formulações após 0, 28, 60 e 90 dias quando armazenados em temperatura ambiente, ou submetidas a $37^{\circ} \mathrm{C}$ ou $45^{\circ} \mathrm{C}$.

\begin{tabular}{|c|c|c|c|c|c|c|c|c|c|c|c|c|}
\hline \multirow[b]{3}{*}{$\begin{array}{l}\text { Formulation } \\
\text { Formulação }\end{array}$} & \multicolumn{12}{|c|}{ Minimum apparent viscosity (cP)/ Viscosidade aparente mínima (cP) } \\
\hline & \multicolumn{4}{|c|}{ Ambient/ Ambiente } & \multicolumn{4}{|c|}{$37^{\circ} \mathrm{C}$} & \multicolumn{4}{|c|}{$45^{\circ} \mathrm{C}$} \\
\hline & T0 & $\mathbf{T} 28$ & T60 & T90 & T0 & T28 & T60 & T90 & T0 & T28 & T60 & T90 \\
\hline V1 & 1605 & 1730 & 1681 & 1520 & 1822 & 2002 & 1868 & 1342 & 1864 & 1503 & 1803 & 2103 \\
\hline $\mathbf{V} 2$ & 1310 & 1425 & 1785 & 1862 & 1183 & 1374 & 1544 & 1726 & 1318 & 1399 & 1688 & 1696 \\
\hline $\mathbf{V 3}$ & 1616 & 1766 & 1935 & 2032 & 1699 & 1682 & 1864 & 1996 & 1690 & 1987 & 1134 & 1849 \\
\hline V4 & 611 & 676 & 560 & 743 & 591.7 & 606.8 & 635.2 & 754.3 & 637.1 & 603 & 782.4 & 742.9 \\
\hline F1 & 1817 & 1754 & 1792 & 1800 & 1785 & 1843 & 1803 & 1520 & 1705 & 1698 & 1495 & 1643 \\
\hline F2 & 1214 & 1367 & 1578 & 1851 & 1248 & 1376 & 1580 & 1792 & 1251 & 1348 & 1395 & 1482 \\
\hline F3 & 1068 & 1098 & 1422 & 1754 & 1093 & 1255 & 1433 & 1743 & 1216 & 1342 & 1427 & 1546 \\
\hline F4 & 686 & 508 & 677 & 887 & 567.1 & 678.6 & 720.2 & 926.3 & 506.6 & 580.3 & 627.6 & 1008 \\
\hline
\end{tabular}

The rheograms showed that the formulations presented tixotropy. The hysteresis area provides information related to the ability of a formulation to restructure after the application of a force. If a formulation presents a high hysteresis area, that indicates an ideal restructure time. There is also an ideal time to formulation reestablishment. It should not be extended in order to avoid excessive flow, however, the time also cannot be too short. The flow over the skin is always followed by the reduction of interaction between the film formed by the formulation and the skin ${ }^{[14 ; 15]}$.

A
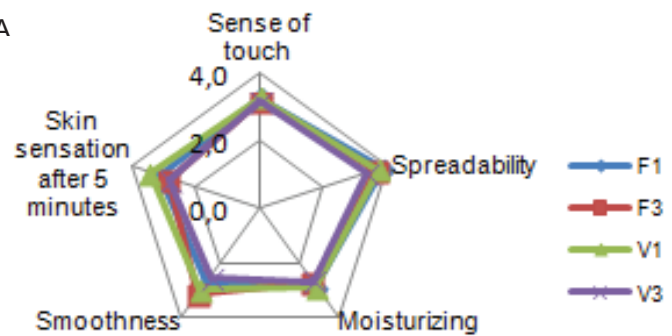

Os reogramas mostraram que as formulações apresentaram tixotropia. A área de histerese contém informações relacionadas com a habilidade de uma formulação em reconstruir a sua estrutura após a aplicação de uma força. Se a formulação apresenta um valor alto da área de histerese, isto indica que há o tempo ideal para a sua reconstrução. Também há um tempo ideal para o reestruturamento de uma formulação, que não deve ser prolongado para evitar um fluxo excessivo e por outro lado, não pode ser curto também. O fluxo sobre a pele tem que ser sempre seguido pela redução de interação entre o filme formado pela formulação e a pele ${ }^{[14 ; 15]}$.

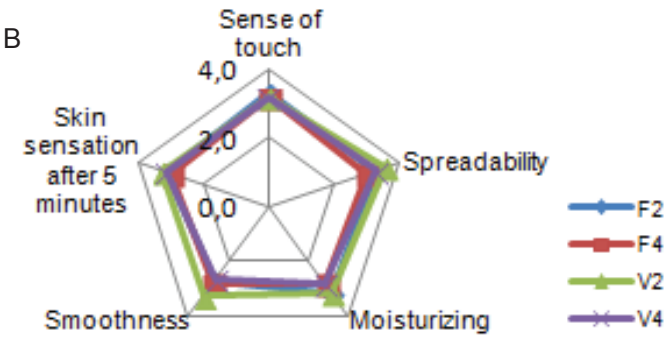

Figure 2/ Figura 2. Sensory analysis of formulations F1, F3, V1 and V3 (A); F2, F4, $\mathrm{V} 2$ and $\mathrm{V} 4(\mathrm{~B})$.

Análise sensorial das formulações F1, F3, V1 e V3 (A); F2, F4, V2 e V4 (B). 
The sensory analysis data (Fig. 2) showed that the formulations were well evaluated in the sensory parameters. The formulations V2 and F2 presented the best sensory profile in some evaluated parameters, such as spreadability, smoothness and skin moisturizing, and these effects were more pronounced when formulations were supplemented with hydrolysed Manihot esculenta tuber extract.

This type of test has a great importance in the development of a cosmetic product, as the sensorial characteristics can influence consumers' acceptance in the choice of a product ${ }^{[16]}$. In this way, the sensory analysis, along with the correct experimental conduct, can promote the success of a cosmetic product ${ }^{[17]}$, fundamentally pleasing to consumers by presenting a differentiated sensory profile ${ }^{[14]}$.

Gel formulations based on Polyacrylamide (and) C1314 Isoparaffin (and) Laureth-7 presented the most agreeable sensory properties and were stable considering the physical stability tests performed, demonstrating that it is an appropriate vehicle for formulations containing hydrolysed Manihot esculenta tuber extract.

\section{Conclusion}

In summary, the formulations developed were stable and showed good sensory properties to improve the skin smoothness and hydration. The gel formulation containing Polyacrylamide (and) C13-14 Isoparaffin (and) Laureth-7 presented the best results in the evaluated parameters, and is the studied vehicle more appropriate for cosmetic formulations containing hydrolysed Manihot esculenta tuber extract.

\section{Conflict of interest}

The author states that there are no financial or personal relationships that might be perceived as potential conflict of interests.
A análise sensorial (Figura 2) mostrou que as formulações foram bem avaliadas nos parâmetros sensoriais. As formulações V2 e F2 apresentaram o melhor perfil sensorial entre os parâmetros avaliados, como espalhabilidade, suavidade e hidratação da pele, e esses efeitos foram mais pronunciados quando adicionados com o extrato hidrolisado da raíz de Manihot esculenta.

Esse tipo de teste tem grande importante no desenvolvimento de um produto cosmético, uma vez que as característica sensoriais podem influenciar na aceitação dos consumidores na escolha de um produto ${ }^{[16]}$. Deste modo, a análise sensorial, junto com a conduta experimental correta, pode promover o sucesso de um produto ${ }^{[17]}$, o que é fundamental para agradar os consumidores, apresentando um perfil sensorial diferenciado ${ }^{[14]}$. As formulações em Gel baseadas em Polyacrylamide (and) C13-14 Isoparaffin (and) Laureth-7 apresentaram o perfil sensorial mais agradável e estáveis considerando os testes de estabilidade física realizados, mostrando que é um veículo apropriado para formulações contendo o extrato hidrolizado da raíz de Manihot esculenta.

\section{Conclusão}

Em conclusão, as formulações desenvolvidas foram estáveis e demonstraram boas propriedades sensoriais, melhorando a sensação de maciez e hidratação da pele. Dentre as formulações estudadas, a formulação em gel contendo Polyacrylamide (and) C13-14 Isoparaffin (and) Laureth-7 foi a que apresentou melhores resultados nos parâmetros avaliados, e é o veículo estudado mais apropriado para formulações cosméticas contendo Extrato hidrolisado da raíz de Manihot esculenta.

\section{Conflito de interesses}

A autora declara que não existem relações financeiras ou pessoais que puderam ser vistas como um potencial conflito de interesses. 


\section{References / Referências}

[1] Isik B, Gurel MS, Erdemir AT, Kesmezacar O. Development of skin aging scale by using dermoscopy. Skin Research and Technology, 2013; 19: 69-74.

[2] Ganceviciene R. et al. Skin anti-aging strategies. Dermato-Endocrinology, 2012. 4. 3. 308-319.

[3] Ryu HS, Joo YH, Kim SO, Park KC, YOUN SW. Influence of age and regional differences on skin elasticity as measured by the Cutometer ${ }^{\circledR}$, Skin Research and Technology, 2008; 14:354-358.

[4] Hirata LL, Sato MEO, Santos CAM. Radicais Livres e o Envelhecimento Cutâneo. Acta Farm. Bonaerense, 2004; 23 (3): 41824.

[5] Jachowicz J, McMullen R, Prettypaul D. Alteration of skin mechanics by thin polymer films, Skin Research and Technology, 2008; 14: 312-319.

[6] Anconi GL, Dal'Belo SE, Silva MM, Camargo Jr FB, Maia Campos PMBG. Evaluation of Sensorial Effects of Gels Containing L-Fucose and L-Rhamnose and its Stability When Associated or Not With Dimethylaminoethanol (DMAE), Wheat Protein or Kinetin in Liposomes. $1^{\circ}$ SINPOSPq (Ribeirão Preto); 2004. CD-ROM.

[7] SILAB. Enginering natural active ingredients. Instensyl®. Technical Information, 2011, France.
[8] Leonardi GR, Maia Campos PMBG. Estabilidade de formulações cosméticas. Inter. J. Pharma, 2001. 3. 4. 154-156.

[9] Capitani RD, Mercurio DG, CAMARGO, F.B.J.; MAIA CAMPOS, P.M.B.G. Stability and clinical efficacy of moisturizing cosmetic formulations containing vitamins $\mathrm{C}$ and E. Biomedical and Biopharmaceutical Research; 2012. 9. 2: 215-224.

[10] Gianeti MD, Gaspar LR, Camargo Jr, FB, Maia Campos PMBG. Benefits of combinations of vitamin $\mathrm{A}, \mathrm{C}$ and $\mathrm{E}$ derivatives in the stability of cosmetic formulations. Molecules, 17 (2012) 2219-30.

[11] Wakemaker TAL, Rosado C, Andrade JP, Fernandes AS, Rijo P, Maia Campos PMBG, Rodrigues LM. Evaluation of the sensory properties of a cosmetic formulation containing green coffee oil. Biomedical and Biopharmaceutical Research; 2013 (10) 1: 101-108.

[12] Gaspar LR, Maia Campos PMBG. Rheological behavior and the SPF of sunscreens. Inter. J. Pharma., 2003. v.250, n.1, p.35-44.

[13] Martin A, Bustamante P, Chun, AHC. Rheology. In: MARTIN, A.; BUSTAMANTE, P.; CHUN, A. H. C. Physical Pharmacy. 4th ed, Philadelphia: Lea \& Febiger., 1993, cap.17, p.453-473.
[14] Camargo Júnior, FB, Bertucci SM, Santos I, Gaspar LR, Maia Campos, PMBG. Stability, Efficacy and Skin Compatibility of Cosmetic Formulations Containing polysaccharides of Plant or Biotechnological Origin. In: 25th IFSCC CONFERENCE, 2009, Melbourne. 25th IFSCC CONFERENCE, 2009.

[15] Dahms GH. Rheology-ita effect on physical SPFs. Soap. Perfum. Cosm., 1996. 69(3), 23-25.

[16] Gomes AL, Langer CM, Oliveira EC, Vairoletto L. Diferentes tipos de pele: diferentes necessidades cosméticas. In: CONGRESSO NACIONAL DE COSMETOLOGIA, 12, 1998. São Paulo, Anais. São Paulo, 1998. p. $220-231$

[17] Tachinardi F, Sá Dias TC, Picirili CAD, Gomes AL, Arruda R. Avaliação sensorial de produtos cosméticos em pele no. In: XVII COLAMIQC, 2005, Cusco CD-ROM. 